\title{
Reduction and Escalation in the Dose of Sunitinib Were Adequately Effective against Gastrointestinal Stromal Tumor of the Small Intestine
}

\author{
Misato Ogata ${ }^{1}$, Hironaga Satake ${ }^{1,2}$, Takatsugu Ogata ${ }^{1}$, Yukimasa Hatachi ${ }^{1}$, Shigeo Hara ${ }^{3}$, \\ Seiichi Hirota ${ }^{4}$ and Hisateru Yasui ${ }^{1}$
}

\begin{abstract}
:
We herein report the first case in which an escalated dose of sunitinib was effective, even after dose reduction. A 64-year-old man with gastrointestinal stromal tumor of the small intestine discontinued adjuvant imatinib because of interstitial pneumonia. After two years, peritoneal recurrence was detected. Sunitinib was started at $50 \mathrm{mg} /$ day for 4 weeks every 6 weeks, after which the dosage was reduced to $37.5 \mathrm{mg} /$ day because of grade 1 gastritis, stomatitis, and a fever. Four months later, computed tomography showed progressive disease. As the adverse events were well-controlled by medication, we escalated the dose to $50 \mathrm{mg} / \mathrm{day}$ and achieved a partial response.
\end{abstract}

Key words: gastrointestinal stromal tumor, sunitinib, dose modification

(Intern Med 58: 3243-3246, 2019)

(DOI: 10.2169/internalmedicine.2806-19)

\section{Introduction}

Gastrointestinal stromal tumor (GIST) is the most common mesenchymal tumor of the gastrointestinal tract. Surgical resection is the first recommended therapy, and systemic chemotherapy is administered for unresectable tumors, as they are resistant to radiotherapy. However, conventional chemotherapies are not applicable, and tyrosine kinase inhibitors (TKIs) show efficacy on GISTs according to the tumor molecular pathogenesis.

Imatinib mesylate, a small molecule that inhibits the activation of KIT and platelet-derived growth factor receptor (PDGFR) $\alpha$ proteins, is the first choice of treatment for GISTs $(1,2)$. Sunitinib has significant clinical benefits in patients with imatinib-resistant GISTs. It is approved at a dose of $50 \mathrm{mg}$ /day for 4 weeks followed by a 2-week off period $(2,3)$, but in a clinical setting, it is usually intolerable due to adverse events. Therefore, dose modification is usually required.
We herein report the first case in which escalating the dose of sunitinib was effective, even after dose reduction. We also show the effectiveness of modifying the sunitinibadministration schedule.

\section{Case Report}

A 64-year-old man visited our hospital with complaints of anorectal pain and incomplete stool evacuation. Computed tomography (CT) and magnetic resonance imaging showed a mass in the left lower quadrant of the abdomen (Fig. 1). As colonoscopy revealed no lesions, a submucosal tumor was suspected. Laparotomy was performed for diagnostic treatment, and the tumor turned out to be a 7.5-cm GIST of the small intestine with a mitotic rate of 23 mitoses per 50 high-power fields (HPF). It harbored a mutation in KIT exon 11 (Fig. 2). The tumor was classified as having a high risk of recurrence using the modified-Fletcher classification, and adjuvant therapy with imatinib was planned. Due to interstitial pneumonia, imatinib was discontinued after eight

\footnotetext{
${ }^{1}$ Department of Medical Oncology, Kobe City Hospital Organization Kobe City Medical Center General Hospital, Japan, ${ }^{2}$ Cancer Treatment Center, Kansai Medical University Hospital, Japan, ${ }^{3}$ Department of Pathology, Kobe City Hospital Organization Kobe City Medical Center General Hospital, Japan and ${ }^{4}$ Department of Surgical Pathology, Hyogo College of Medicine, Japan

Received: February 6, 2019; Accepted: June 10, 2019; Advance Publication by J-STAGE: July 22, 2019

Correspondence to Dr. Hironaga Satake, takeh1977@gmail.com
} 
months of administration.

After a treatment-free period of about two years, peritoneal recurrence was detected (Fig. 3). This time, sunitinib at $50 \mathrm{mg} /$ day was introduced because imatinib was intolerable due to interstitial pneumonia. However, grade 1 gastritis, grade 1 stomatitis, and a grade 1 fever were subsequently observed, leading to a dose reduction to $37.5 \mathrm{mg} /$ day. A schedule of 4 weeks on and 2 weeks off with $37.5 \mathrm{mg}$ resulted in a partial response after approximately two months. Concurrently, varicella zoster virus infection occurred. He also suffered peripheral nerve disorder. As we realized that the dose intensity could not be maintained by the current approach of drug administration, the sunitinib schedule was modified to two weeks on and one week off.

After two months, CT showed disease progression. Changing the drug to regorafenib was a reasonable choice, but sunitinib escalation was also feasible, as the adverse events were not observed at that time. In fact, prophylaxis against gastritis, stomatitis, and hand-foot syndrome was effective. As the patient did not want to change to another drug, the dose of sunitinib was escalated to $50 \mathrm{mg} /$ day for 2 weeks followed by 1 week off. This time, the patient exhib-

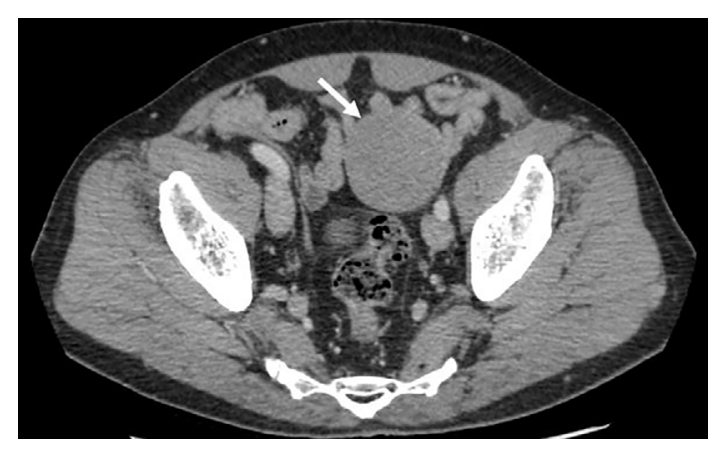

Figure 1. Computed tomography (CT) image obtained before surgery. A round mass (white arrow) in the left lower quadrant of the abdomen was detected. ited a partial response (Fig. 4). The patient has continued sunitinib for 20 months now, and the tumor remains small, although dose reduction to $25 \mathrm{mg}$ /day was required because of grade 3 hypertension and grade 2 edema (Fig. 5, 6).

\section{Discussion}

We experienced a case, in which an escalation in the dose of sunitinib, even after dose reduction, was effective against GIST.

GISTs exhibit a varying degree of malignancy potential, and risk stratification is important when considering the treatment strategy. One of the most popular risk-stratification approaches is the modified-Fletcher classification (4). According to this system, our case, whose tumor was classified as high-risk because of a mitotic index of $>10$ per 50 HPFs, has a $15-20 \%$ risk of recurrence and is recommended to receive adjuvant imatinib for 3 years (5). Furthermore, GISTs usually harbor activating mutations in either KIT (75-80\%) or PDGFR $\alpha$ (5-10\%) (6), and the genotype has been identified as a predictor of the response to imatinib. In the present case, a mutation at KIT in exon 11 was detected, implying a better response to imatinib (7).

For recurrent and unresectable GISTs, the first recommended TKI is imatinib. However, some patients show primary or early resistance to imatinib, and acquired resistance develops after a median of approximately two years of treatment. In case of resistance, sunitinib, an oral multitargeted receptor TKI, is the next choice. Sunitinib and imatinib are similar in that they both bind within the ATP-binding domain of KIT and PDGFRs, but the significant difference between the two is that sunitinib inhibits VEGFR kinases, which are important in tumor-related angiogenesis, whereas imatinib cannot inhibit them. This is thought to be the main reason underlying the clinical benefit of sunitinib for imatinib-resistant GISTs (3). Considering these mechanisms, imatinib-intolerable patients, such as the patient in the pre-

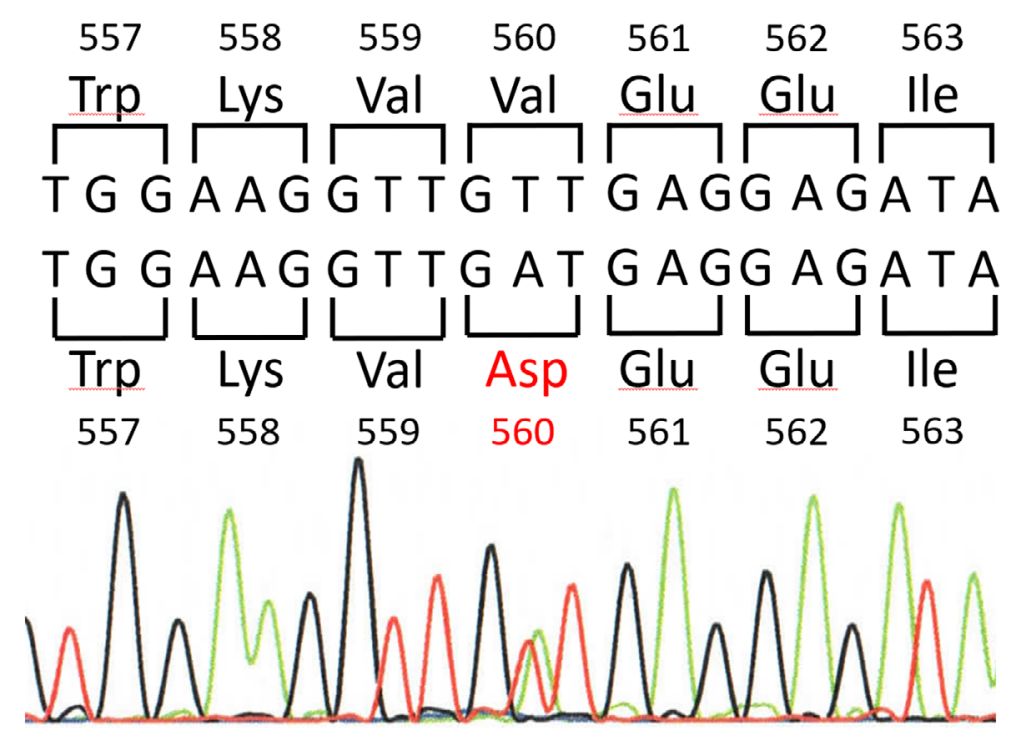

Figure 2. The gene mutation detected in this tumor. 

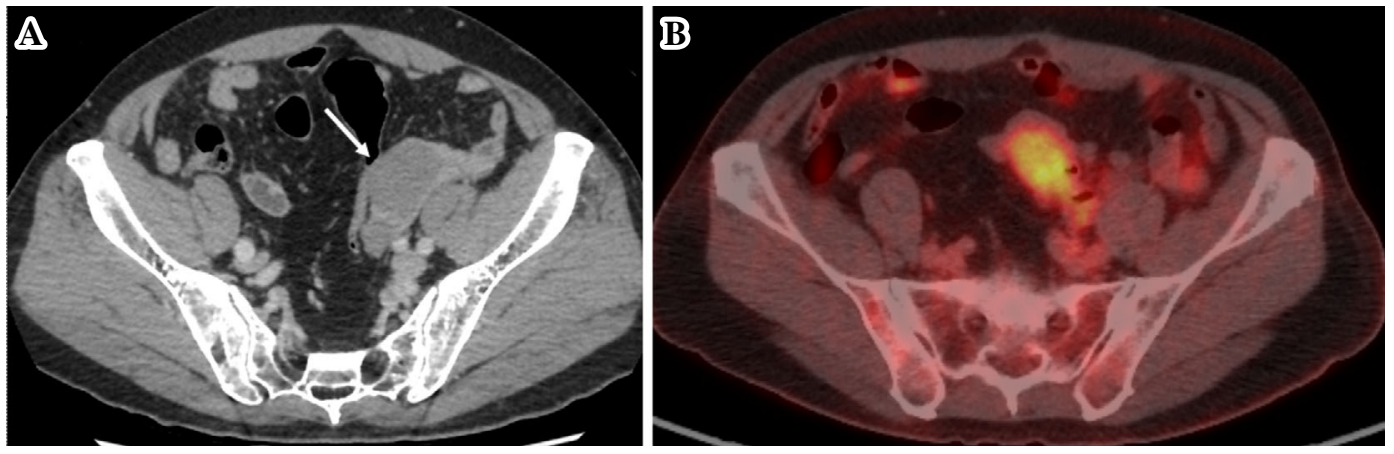

Figure 3. Intrapelvic recurrence lesions revealed by CT (A, indicated with white arrow) and positron emission tomography (PET, B).

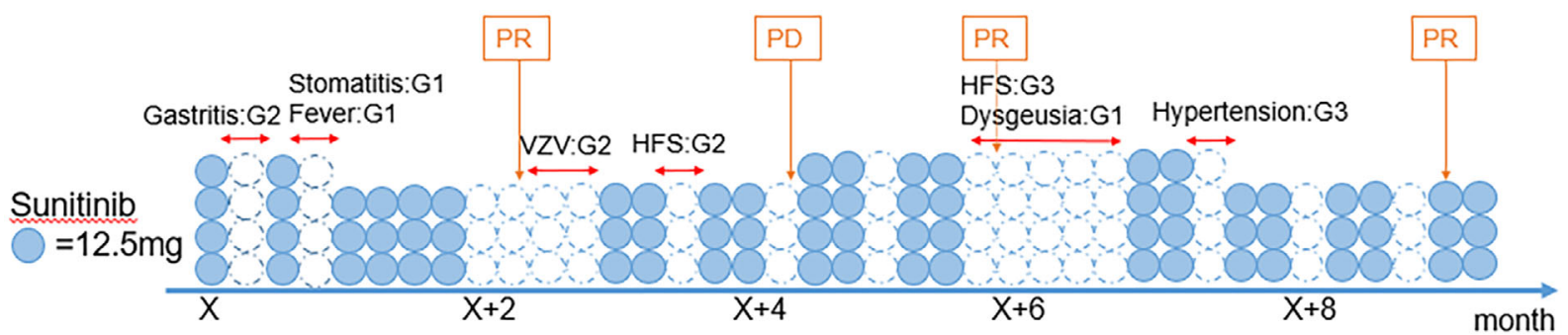

Figure 4. Treatment progress chart.

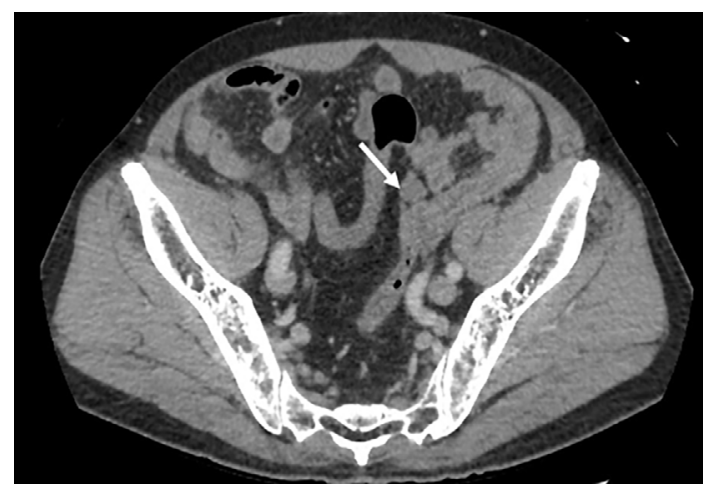

Figure 5. Latest CT image taken 17 months after starting the administration of sunitinib. The intrapelvic lesions indicated with a white arrow decreased in size, and the lesions around the rectum diminished as well.

sent study, can also be treated with sunitinib.

Sunitinib is recommended to be used at a dose of $50 \mathrm{mg} /$ day for 4 weeks followed by a 2-week off period according to a phase I trial (8). However, Japanese post-marketing surveillance has reported that as many as $60 \%$ of patients with GIST require dose reduction (9), so modification of the drug dose and treatment schedule is usually needed. To our knowledge, the present case is the first to show the effectiveness and safety of escalating the sunitinib dose even after a previous dose reduction. In addition to escalation, modifying the treatment schedule might also be effective. Preclinical data have reported tumor growth during the offdosing period, suggesting that sunitinib might be effective when used continuously (10). Furthermore, a phase II trial

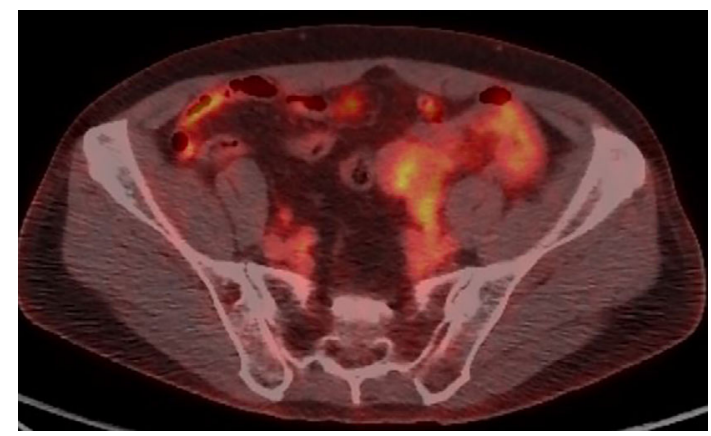

Figure 6. Latest PET image taken 18 months after starting the administration of sunitinib. The FDG uptake in the tumor decreased.

showed that the concentration of sunitinib decreased to predose levels during a 14-day rest period (11). Based on these reports, various modifications of sunitinib administration have been considered. For example, a phase I trial of sunitinib for two weeks followed by a one-week off period (2/1 schedule) has shown tolerability among patients. This $2 / 1$ schedule kept the sunitinib concentration detectable even on day 1 of course 2, while no significant drug accumulation between courses was detected. This indicates that the $2 / 1$ schedule can prolong sunitinib exposure without inducing intolerance (12).

Kinase genotype has also been shown to influence the clinical activity of sunitinib. In detail, the progression-free survival (PFS) is known to be shorter (5.1 to 7.0 months) for patients with primary KIT exon 11 mutations than for those with KIT exon 9 mutations or with a wild-type geno- 
type $(13,14)$. It is difficult to compare the PFS of the present case with the values in other cases because our patient used sunitinib as the first-line treatment due to intolerance of imatinib. However, we can safely say that sunitinib was effective in our case.

Supplying sufficient medication is also believed to be important, as demonstrated in this case; whereby a proton pump inhibitor against gastritis, topical steroids against stomatitis and hand-foot syndrome, and an Angiotensin Converting Enzyme inhibitor and calcium channel blocker against hypertension were administered.

In conclusion, the present findings suggest that escalation of sunitinib can be considered after reducing the dose due to adverse events and that the treatment schedule can be modified when required.

The authors state that they have no Conflict of Interest (COI).

\section{References}

1. Demetri GD, von Mehren M, Blanke CD, et al. Efficacy and safety of imatinib mesylate in advanced gastrointestinal stromal tumors. N Engl J Med 347: 472-480, 2002.

2. Network NCC. Soft Tissue Sarcoma Ver2. 2018 [Internet]. [cited 2018 Dec 31]. Available from: https://www.nccn.org/professionals/ physician_gls/pdf/sarcoma.pdf

3. Demetri GD, van Oosterom AT, Garrett CR, et al. Efficacy and safety of sunitinib in patients with advanced gastrointestinal stromal tumour after failure of imatinib: a randomised controlled trial. Lancet 368: 1329-1338, 2006.

4. Joensuu H. Risk stratification of patients diagnosed with gastrointestinal stromal tumor. Hum Pathol 39: 1411-1419, 2008.

5. Joensuu H, Eriksson M, Sundby Hall K, et al. One vs three years of adjuvant imatinib for operable gastrointestinal stromal tumor: a randomized trial. JAMA 307: 1265-1272, 2012.

6. Rubin BP, Heinrich MC, Corless CL. Gastrointestinal stromal tu- mour. Lancet 369: 1731-1741, 2007.

7. Heinrich MC, Owzar K, Corless CL, et al. Correlation of kinase genotype and clinical outcome in the North American Intergroup Phase III Trial of imatinib mesylate for treatment of advanced gastrointestinal stromal tumor: CALGB 150105 Study by Cancer and Leukemia Group B and Southwest Oncology Group. J Clin Oncol 26: 5360-5367, 2008.

8. Faivre S, Delbaldo C, Vera K, et al. Safety, pharmacokinetic, and antitumor activity of SU11248, a novel oral multitarget tyrosine kinase inhibitor, in patients with cancer. J Clin Oncol 24: 25-35, 2006.

9. Komatsu Y, Ohki E, Ueno N, et al. Safety, efficacy and prognostic analyses of sunitinib in the post-marketing surveillance study of Japanese patients with gastrointestinal stromal tumor. Jpn J Clin Oncol 45: 1016-1022, 2015.

10. Abrams TJ, Murray LJ, Pesenti E, et al. Preclinical evaluation of the tyrosine kinase inhibitor SU11248 as a single agent and in combination with "standard of care" therapeutic agents for the treatment of breast cancer. Mol Cancer Ther 2: 1011-1021, 2003.

11. Houk BE, Bello CL, Poland B, Rosen LS, Demetri GD, Motzer RJ. Relationship between exposure to sunitinib and efficacy and tolerability endpoints in patients with cancer: results of a pharmacokinetic/pharmacodynamic meta-analysis. Cancer Chemother Pharmacol 66: 357-371, 2010.

12. Britten CD, Kabbinavar F, Hecht JR, et al. A phase I and pharmacokinetic study of sunitinib administered daily for 2 weeks, followed by a 1-week off period. Cancer Chemother Pharmacol 61: 515-524, 2008.

13. Heinrich MC, Maki RG, Corless CL, et al. Primary and secondary kinase genotypes correlate with the biological and clinical activity of sunitinib in imatinib-resistant gastrointestinal stromal tumor. J Clin Oncol 26: 5352-5359, 2008.

14. Reichardt P, Demetri GD, Gelderblom H, et al. Correlation of KIT and PDGFRA mutational status with clinical benefit in patients with gastrointestinal stromal tumor treated with sunitinib in a worldwide treatment-use trial. BMC Cancer 16: 22, 2016.

The Internal Medicine is an Open Access journal distributed under the Creative Commons Attribution-NonCommercial-NoDerivatives 4.0 International License. To view the details of this license, please visit (https://creativecommons.org/licenses/ by-nc-nd/4.0/).

(C) 2019 The Japanese Society of Internal Medicine Intern Med 58: 3243-3246, 2019 